\title{
La pose de l'épicier. Reportage ethnographique dans les épiceries grecques et turques de Bruxelles
}

The grocer's pose. Ethnographic reportage in the Greek and Turkish groceries of Brussels

Katerina Seraïdari

\section{OpenEdition}

Journals

Édition électronique

URL : http://journals.openedition.org/itti/1005

DOI : 10.4000/itti. 1005

Éditeur

Université de Poitiers

\section{Référence électronique}

Katerina Seraïdari, «La pose de l'épicier. Reportage ethnographique dans les épiceries grecques et turques de Bruxelles », Images du travail, travail des images [En ligne], 4 | 2017, mis en ligne le 01 septembre 2017, consulté le 14 avril 2021. URL : http://journals.openedition.org/itti/1005 ; DOI : https://doi.org/10.4000/itti.1005

Ce document a été généré automatiquement le 14 avril 2021

Images du travail, travail des images 


\section{La pose de l'épicier. Reportage ethnographique dans les épiceries grecques et turques de Bruxelles}

The grocer's pose. Ethnographic reportage in the Greek and Turkish groceries of Brussels

Katerina Seraïdari

1 Dans un article qui pose la question de la représentabilité du travail, Philippe Leroux $(1996,132)$ constate que «bien des activités du commerce, grand ou petit, semblent manquer au répertoire». Cette présentation, qui analyse la manière dont la photographie met en scène le travail de l'épicier, vise à combler, autant que faire se peut, ce vide. L'épicerie n'est pas peuplée de machines impressionnantes; elle n'est pas un symbole de la modernité, à l'inverse du supermarché - son grand rival ; elle n'est pas non plus un vestige du passé, puisqu'elle est remplie des marchandises dernier cri. Contrairement à l'usine et à la mine qui sont des environnements inhospitaliers, l'épicerie est un espace accueillant. Tandis que l'usine ou la mine constituent des mondes spectaculaires, peu connus, inaccessibles et étranges qui ont maintes fois retenu l'attention des photographes, l'épicerie reste un lieu banal, insignifiant et familier. Ce n'est pas un lieu dépaysant « qui porte à regarder» (Bourdieu, 1965, 57); c'est un espace qu'il faut arracher « au rapport de familiarité inattentive avec le monde quotidien » (ibid., 59). Cette familiarité rend le travail effectué dans l'épicerie encore plus invisible et insaisissable.

Deux types de matériaux seront mobilisés, ethnographiques et photographiques. Les enquêtes ethnographiques ont débuté en septembre 2009 afin d'étudier, dans une premier temps, les relations entre les communautés grecque et turque de Bruxelles (Seraïdari, 2012); et dans un deuxième temps, les épiceries grecques et turques (Seraïdari, 2011, 2011-2012 et 2016). En collaboration avec le photographe Christophe Delory, quinze épiceries bruxelloises ${ }^{1}$ ont été photographiées en juin 2013. Ces commerces de proximité ${ }^{2}$ qui s'appuient sur une main-d'œuvre familiale, sont indépendants juridiquement, sans aucune attache à des chaînes ou des franchises. 
Tandis qu'aucune épicerie grecque n'emploie $d^{\prime}$ " ouvriers $»^{3}$, certaines épiceries turques le font, même si leur nombre ne dépasse jamais les trois employés. Si certaines d'entre elles proposent des produits liés à l'origine du gérant ou sont caractérisées par une décoration évoquant le pays d'origine, leur fonctionnement est tout à fait similaire à celui des magasins tenus par des Belges.

3 Ce projet photographique, conçu comme un reportage ethnographique, avait pour ambition d'aller au-delà de la fonction purement illustrative de la photographie ; il ne constituait donc ni une simple étape dans la collecte de données ethnographiques ni un simple support à l'entretien (Conord, 2007, 16-18). La photographie non seulement facilite mais aussi permet d'approfondir l'observation, car elle retient des détails et des éléments non perceptibles immédiatement ou qui paraissent secondaires au premier abord. Elle élargit la connaissance et la sensibilité de l'ethnologue concernant ce lieu de travail et de sociabilité, les personnes qui le fréquentent et les objets (décoratifs ou marchands) qui y sont exposés et vendus. La photographie correspond tant à une intention d'exactitude ethnographique qu'à une volonté conservatrice : en saisissant des fragments du présent, elle devient un témoin qui permet de conserver les traces des transformations urbaines, surtout dans ce cadre marchand dont la réalité est fluctuante, puisque ces magasins changent souvent de propriétaires et de décoration.

D'un point de vue méthodologique, les matériaux ethnographiques ${ }^{4}$ concernent vingtune épiceries, auxquelles viennent s'ajouter cinq autres épiceries considérées comme «périphériques» (et pour lesquelles les données recueillies sont moins approfondies). Mon immersion prolongée dans cet univers marchand a pris une nouvelle tournure avec l'intervention de Christophe Delory, qui a su imposer son autonomie de regard. Notre projet nous a forcé à croiser deux points de vue, celui du photographe (qui, en tant que faiseur d'images, veut fixer l'instant) et celui de l'ethnologue (qui, en tant qu'enregistreur de paroles échangés et de récits de vie, s'inscrit dans la durée). Ces deux lectures complémentaires permettent de mieux saisir les différentes échelles de la « temporalité commerçante ».

L'acte photographique est défini par trois temps :

a. la phase préparatoire afin de circonscrire et de sélectionner l'objet à photographier : ce qui implique des critères comme la représentabilité, la diversité ou la singularité

b. la prise de vue

c. la période définie par la production et l'utilisation de l'objet-photographie ${ }^{5}$.

6 La période préparatoire a été nécessaire pour s'assurer de l'accord des épiciers. Un seul épicier a envisagé notre projet avec méfiance, nous soupçonnant de vouloir ouvrir un commerce et «voler» ses idées sur la manière de ranger les produits. Dans ce cas, notre démarche photographique lui semblait motivée par des intentions commerciales masquées : elle était bel et bien perçue comme un projet de connaissance mais qui ne serait pas liée à des fins artistiques ou scientifiques ${ }^{6}$. Deux autres épiciers ont refusé d'être photographiés, l'un parce que son magasin était en travaux et l'autre parce que, comme elle me l'a dit :

«Je n'aime pas les photos. Je ne les regarde jamais, je n'ai pas d'album, pour ne pas penser à ceux qui ne vivent plus. Je n'aime pas les photos depuis la mort de ma mère, elle avait juste cinquante ans, il y a trente-deux ans déjà ».

7 Dans ce cas, un deuil personnel a conditionné le rapport négatif de l'épicière à la photographie. Enfin, un troisième épicier a refusé, parce que son commerce se trouvait près de l'Université et il avait comme principe de décliner toute sollicitation pour des 
travaux universitaires: pendant le déroulement des enquêtes ethnographiques cet obstacle n'a pas été évoqué et c'est l'indiscrétion de l'acte photographique qui a probablement déterminé son refus.

Si la phase préparatoire donne une nouvelle orientation aux relations entre l'ethnologue et ses interlocuteurs, le moment de la prise photographique implique un troisième acteur, le photographe. Pour paraphraser Pierre Lannoy (2010, 127-128), ce n'est pas l'épicerie qui se met en scène, c'est le photographe qui la transforme en studio, dans lequel les travailleurs continuent d'exécuter les gestes habituels de leur travail. L'intervention du photographe crée une temporalité spécifique, un «hors temps » qui interrompt la routine quotidienne. Associée à un matériel technique sophistiqué, la présence d'un producteur d'images transforme les épiciers en "star de cinéma ", comme le révèlent les plaisanteries qu'ils échangeaient avec les clients pendant les prises : évocation d'Hollywood ou de magazines comme Playboy, références à la une de journaux qui leur serait dédiée et à Photoshop ${ }^{7}$ qui pourrait corriger d'éventuelles imperfections. D'autre part, la prise photographique change la qualité des lieux : certains clients ont assimilé une épicerie turque de Saint-Gilles ou une épicerie grecque d'Anderlecht à des «monuments historiques" qui méritaient d'être photographiés, du fait de leur ancienneté dans le quartier. En effet, la prise d'images est, en elle-même, un acte qualifiant ce qui est mémorable, ce qui mérite l'attention ; elle correspond à un acte de reconnaissance de l'importance que recèle ce moment, cette personne ou cet endroit particulier. Ce processus a donc un impact performatif : l'appareil photographique attribue au sujet qu'il cible une valeur nouvelle, une signification valorisante. Toutefois, nous avons essayé de nous « en tenir à la recherche d'invisibilité (règle d'interférence minimale), à l'adoption d'un point d'observation neutre » (Colleyn, 1999, 37).

Étant donné que pour chacune des quinze épiceries entre trente et cinquante clichés ont été sélectionnés par le photographe comme "montrables ", il devient évident que seule une toute petite partie de ce corpus sera ici soumise à l'analyse iconographique. Ils n'ont pas été choisis par rapport à leur valeur artistique ou plastique mais pour leur lisibilité. Si le but était de montrer dans quelle mesure l'épicerie fonctionne comme lieu de sociabilité, d'autres photographies auraient été choisies; en l'occurrence, celles qui révèlent de manière claire les différentes séquences de la banalité du travail ont été privilégiées. Dans la première section, nous verrons comment la photographie saisit tant les aspects techniques que relationnels du travail de l'épicier. La deuxième section examinera dans quelle mesure la photographie parvient à révéler les différents statuts sociaux dans ce lieu de travail. La troisième section montrera comment la photographie nous permet de passer des espaces extérieurs et publics aux espaces intérieurs et privés, qui sont par définition moins «montrables ». La quatrième section, en guise de conclusion, reviendra sur le rapport entre l'analyse ethnographique et l'analyse iconographique.

Dans cet article, quelques épiciers grecs et turcs de Bruxelles seront érigés en représentants d'un groupe professionnel dans son ensemble : en faisant abstraction de leurs spécificités personnelles, nationales et confessionnelles, nous laisserons leurs photographies parler de leur activité et de leurs pratiques professionnelles. De la même manière que les photographies familiales constituent un compromis entre la représentation d'une famille idéale (à laquelle les personnes qui prennent la pose essaient de se conformer) et la réalité de la famille dans laquelle nous vivons, les images 
d'épiciers qui seront au cœur de l'analyse font coïncider le quotidien de ces commerçants bruxellois et nos conceptions de ce qu'est le métier de l'épicier.

\section{La saisie photographique de l'épicier à la caisse}

11 L'activité de l'épicier est définie par des moments d'affluence, durant lesquels il est assailli par des vagues de clients et par des moments plus calmes dont il profite pour ranger ou faire ses commandes. La photographie découpe, ordonne et sépare ces séquences qui se succèdent au cours de la journée. Si cette forme de mise en image ne saisit que le morcellement du travail humain, elle parvient toutefois, par la captation des détails, à représenter les deux pôles de l'activité marchande : les temps creux et les «temps pleins ».

Image 1.

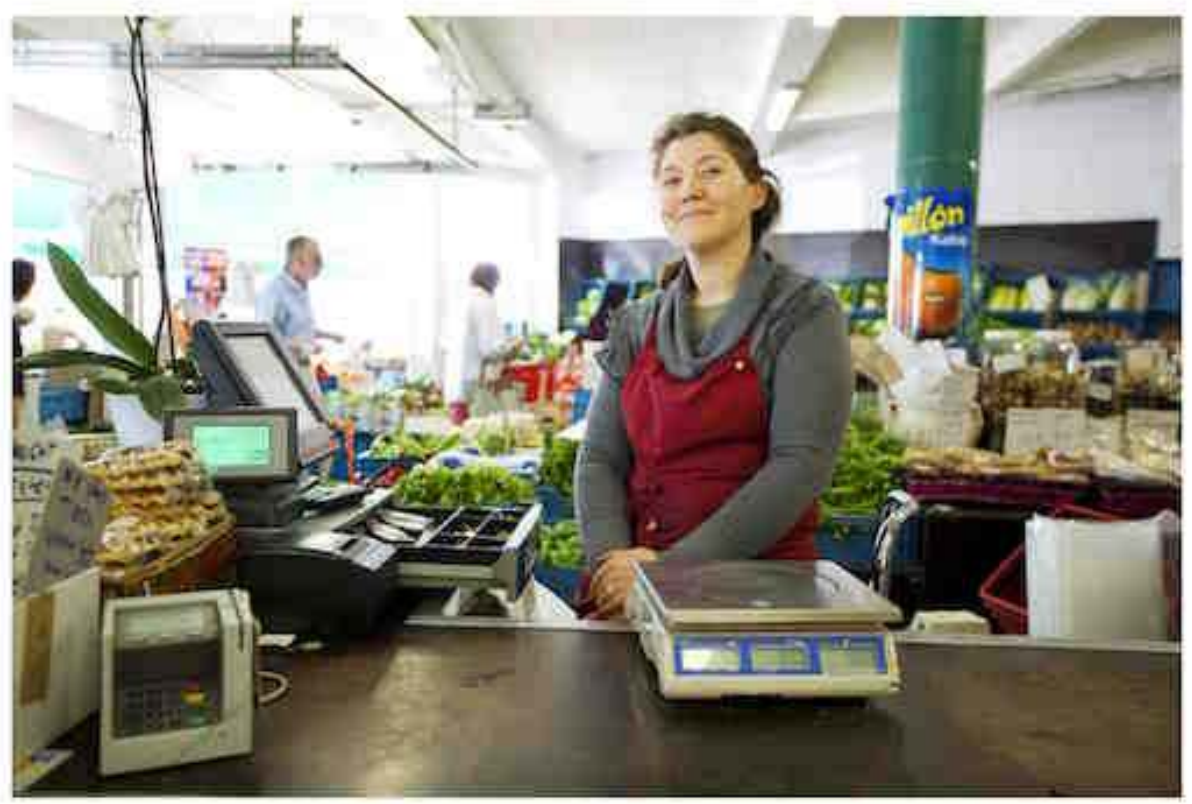

La photographie d'une épicière souriante, en tablier rouge, les mains croisées mais assise devant un tiroir-caisse encore ouvert, montre bien que le temps creux qui permet la pose, a été précédé et sera suivi par des «temps pleins». Le travail est ici arrêté pour le besoin de la photographie et l'épicière fixe fièrement l'objectif tout en gardant sa position de travail. Elle se tient la tête haute et s'impose par son regard. L'axe vertical de la colonne verte à sa droite et le fait qu'elle se trouve au centre des lignes de fuite du plafond soulignent sa position dominante. En arrière plan, on distingue trois clients qui restent flous : un homme, une femme avec un sac à main puis une autre à peine visible à gauche. L'épicière est entourée de ses outils de travail : la balance électronique, la machine pour les paiements par carte de crédit et, surtout, la caisse et son tiroir avec des pièces et des billets méticuleusement rangés. D'ailleurs, dans ce cliché, l'épicière est coupée au niveau des hanches à cause de la caisse.

La caisse structure l'espace du magasin (son positionnement conditionnant le parcours et la circulation des clients), tout en constituant un point de vente important: les 
produits susceptibles de susciter un achat impulsif y sont placés - comme les gaufres et les nougats annoncés par une pancarte, à gauche de l'image. La caisse est toujours gérée par une personne de confiance, le plus souvent un membre de la famille; le cas échéant, l'installation d'une caméra de vidéosurveillance permet au propriétaire de contrôler non seulement les transactions avec les clients (pouvant ainsi faire face à des réclamations de leur part) mais aussi l'employé qui pourrait abuser de la situation. Certains patrons refusent néanmoins de placer une caméra au-dessus de la caisse, pour ne pas insulter celui à qui cette tâche est confiée, surtout quand il s'agit d'un parent, même lointain.

La personne qui a la responsabilité de la caisse gère aussi le crédit : c'est elle qui choisit qui peut bénéficier de ce régime, réservé aux clients les plus fidèles; c'est elle aussi qui peut accorder des réductions ou des faveurs (baisser le prix d'un fruit trop mûr ou peu demandé, offrir un article aux enfants d'une cliente). La caisse constitue le lieu d'échange par excellence entre l'épicier et les acheteurs : c'est ici qu'on échange sur l'actualité politique, la météo, les émissions télé de la veille, les nouvelles du quartier et tous ces sujets de conversation qui fleurissent dans les commerces de proximité. Elle est au centre des relations sociales qui sont nouées dans une épicerie, surtout après la généralisation des courses en libre-service ${ }^{8}$ et la disparition du service personnalisé qui ont marqué le début de l'autonomie du consommateur, jusque-là contraint de se faire servir par l'épicier.

15 Ce cliché met l'épicière en vedette : ayant la caisse comme poste de travail, elle est la maitresse des lieux. Mais la photographie parle aussi de la répartition des rôles sexuels et des tâches qui ne sont pas vraiment interchangeables. Dans la mesure où l'épicerie est, dans une grande majorité de cas, une affaire de couple, la division du travail correspond à une logique assez traditionnelle. Les hommes s'occupent principalement du Marché matinal (étant obligés d'y aller vers 4 h00 ou 5 h00 du matin), des livraisons et de l'approvisionnement; ce sont eux qui portent et qui se chargent des tâches les plus lourdes physiquement. De leur côté, les femmes préparent certains des produits en vente $^{9}$; elles tiennent aussi la caisse, comme le montre ce portrait de la propriétaire du commerce. Immobiles et assises pendant de longues heures, les épicières ont souvent un radiateur à proximité, surtout en hiver, et sont habillées chaudement, comme ici, la photographie ayant pourtant été prise en juin. D'autant plus que la caisse est souvent placée près de la porte d'entrée qui reste ouverte même en hiver. Si la photographie parvient à rendre compte de cette réalité, elle ne montre pas pour autant dans quelle mesure la place de la femme dans ce type d'entreprise familiale a longtemps été fragile et invisible ${ }^{10}$. 
Image 2.

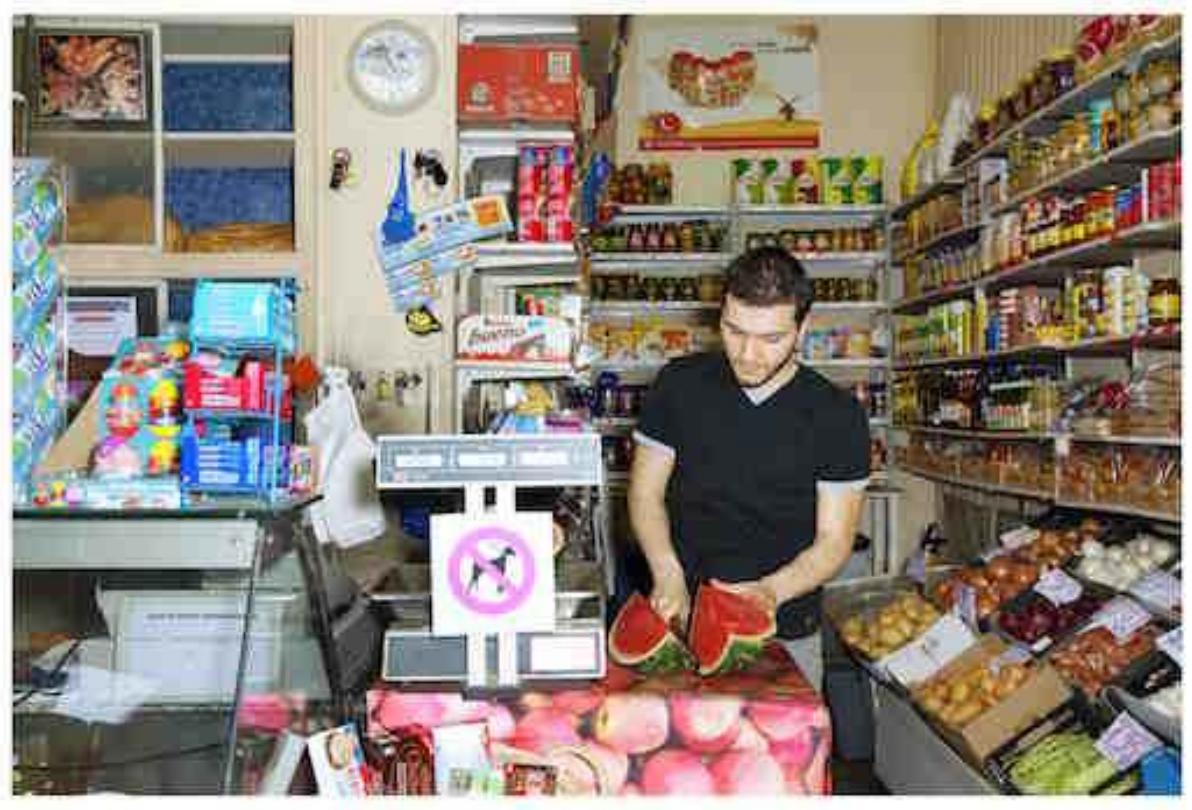

Tandis que la première photographie constitue un portrait, la seconde est prise sur le vif dans une épicerie bien plus exiguë et située dans un quartier populaire. Le jeune épicier est saisi dans une posture de travail qui révèle les limites du self-service. Le fait de couper des fruits pour vendre de petites portions ou dans le cadre d'une dégustation à des fins promotionnelles, renvoie à ce qui reste du service personnalisé. Si le libreservice a généralisé la vente de produits emballés à prix fixes, les clients peuvent encore acheter la quantité exacte qu'ils souhaitent dans certains cas: la moitié ou le quart d'une pastèque mais aussi tant de grammes de semoule, d'olives ou d'autres produits encore vendus en vrac. Dans ce cas précis, le comptoir est transformé en plan de travail. La caisse est placée derrière le réfrigérateur; près d'elle, une calculette, autre outil indispensable de l'épicier. Le client peut ainsi voir le poids affiché sur la balance mais pas le prix affiché sur la caisse, le ticket permettant de compenser ce manque de visibilité. Malgré le manque évident d'espace, le coin est aussi transformé en lieu de tentation pour le client: chewing-gums et friandises sont placés sur le réfrigérateur, dans une corbeille devant le comptoir et sur les étagères derrière la balance.

17 La comparaison avec le cliché précédent permet non seulement de mesurer la distance socio-économique qui sépare les deux commerces mais aussi de contester l'idée trop romantique d'une « communauté de destin ». L'assurance de la femme qui trône dans une épicerie huppée, connue dans toute la ville de Bruxelles pour la qualité de ses fruits et légumes (ce qui la rapproche d'un commerce de produits-bio), contraste avec l'attitude de ce jeune homme, qui tient un magasin beaucoup plus modeste : c'est son père qui le lui a transmis il y a peu de temps. La spécialisation de la première épicerie s'oppose au caractère "généraliste » de la seconde ${ }^{11}$, ce que la photographie parvient à capter par la différenciation entre les produits raffinés proposés près de la caisse dans la première photographie (gaufres et nougats) et leur banalité dans la seconde (Kinder 
Bueno et Mentos). En effet, la caisse en tant que point de vente révèle assez clairement quelle est l'orientation du magasin et à quel public il s'adresse.

Image 3.

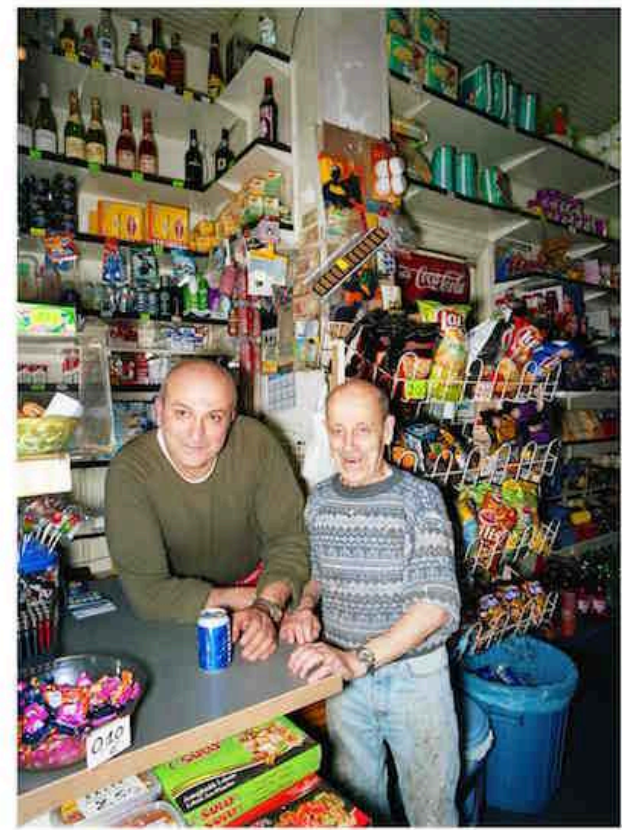

Lieu de vente et plan de travail, la caisse est aussi un espace privilégié de sociabilité. Cette troisième épicerie est également située dans un quartier populaire, comme les produits qui entourent le comptoir (chips, briquets et cigarettes, sucettes et gâteaux orientaux) le laissent deviner. L'épicier pose avec un ami bien plus âgé, à qui une canette vient d'être offerte. Si le tablier constitue un attribut facilement identifiable ${ }^{12}$, celui que le propriétaire porte ici autour de la taille est à peine visible. Toutefois, le pantalon sale de son ami (faisant probablement une pause après le travail) interdit toute confusion entre les deux rôles: l'épicier fait toujours attention à sa tenue vestimentaire, non seulement par déontologie professionnelle, mais aussi à cause des contrôles des services d'hygiène auxquels il est soumis. Un nombre important d'épiciers bruxellois habite derrière ou au-dessus du magasin, ce qui leur permet de changer de vêtements dans la journée, si nécessaire.

Les amis peuvent rester consommer tranquillement leur boisson dans ce commerce, même si l'arrivée de clients interrompt régulièrement l'échange. La poubelle bleue de recyclage, à droite, accueille les canettes vidées sur place. Le cliché saisit une interaction qui dépasse, tout en le redéfinissant, ce cadre précis de travail : la présence décontractée de cet homme âgé transforme le commerçant en hôte qui reçoit un ami et rend confuse la division entre domaine privé et domaine public (question sur laquelle je reviendrai dans la troisième section). De ce point de vue, l'image révèle le côté le moins évident de l'activité commerciale, puisqu'elle nous fait sortir de la logique marchande et nous fait entrer dans l'économie du don. Néanmoins, il n'y a pas que les amis qui 
consomment sur place; certains clients le font aussi, transformant l'épicerie en barcafé, comme les épiciers eux-mêmes le disent en s'amusant.

Selon Monique Peyrière $(2012,67)$, « le travail est à présent filmé d'autant plus dans ses marges, quand le film, à l'usine, préfère la cantine, le dispensaire ou le vestiaire : temps morts, moments de pause ». Il serait pourtant erroné de considérer que les moments de pause dans une épicerie définissent les marges de l'activité commerciale. L'épicier est un professionnel tourné vers le monde matériel des artefacts et des articles à vendre : il doit ranger, exposer, mettre en valeur la marchandise, se heurtant aux contraintes spatiales que le local impose. Mais son travail ne s'arrête pas là ; dans la mesure où il exerce un métier de service à forte composante relationnelle, il a aussi pour mission de servir la clientèle. L'ambiance familiale permet aux commerces de proximité de se démarquer de l'impersonnalité qui règne dans les grandes surfaces; c'est cette qualité d'accueil qui fait d'eux, selon le discours des responsables des politiques urbaines, une valeur à préserver et un élément indispensable de l'équilibre urbain.

L'ambiance en tant que stratégie de vente qui vise à fidéliser la clientèle, ne peut pas être dissociée du tempérament du propriétaire. L'épicier peut devenir un confident et même un "psy" (terme qui revient souvent dans mes enquêtes de terrain) pour certains de ses clients. Toutefois, ses prestations ne sont pas uniformes, dans la mesure où elles dépendent de ses affinités ou de ses intérêts. Dans tous les cas, "une progression du temps de travail relationnel» dans ce contexte n'implique pas forcément une baisse de la "productivité » (Gadrey, 2005, 29). Bien sûr, la question se pose de savoir si le temps passé avec un client fait toujours partie du «travail relationnel ", dans la mesure où le terme «travail » implique déjà une recherche de productivité. Autrement dit, ces échanges relationnels peuvent être situés au-delà du cadre professionnel et de ses buts.

Quant aux services que l'épicier rend, ils ne sont pas seulement d'ordre psychologique. Il peut être sollicité pour aider un compatriote, qui ne maîtrise pas bien la langue française, à régler ses problèmes avec l'administration belge. Il peut sponsoriser certains événements, comme l'élection d'une Miss ou participer à la fête annuelle du quartier, investissant ainsi dans le domaine des loisirs toujours en relation avec la vie du quartier dans lequel le commerce se trouve. De même, il peut surveiller, avec la complicité de la famille du client, les achats de quelqu'un qui a des problèmes d'alcoolisme ou qui vit seul: dans le dernier cas, la famille veut souvent s'assurer que la personne en question se nourrit convenablement et sort se promener régulièrement. L'épicier est l'œil du quartier et peut même prévenir la police, comme cette interlocutrice, dont le commerce est situé dans un quartier aisé : une fois alertée, la police a constaté le décès d'une cliente qui vivait seule et ne faisait plus ses sorties régulières depuis quelques jours.

La sociabilité, avec ses moments de distraction, ne conduit donc pas à un détournement d'usage des lieux ; bien au contraire, en tant que facteur d'attractivité et de rentabilité, elle est constitutive de ce métier. Mais l'épicier n'est pas seulement un acteur économique, un calculateur rationnel des gains que la sociabilité peut lui conférer. Les services qu'il rend peuvent aussi être une source de fierté et de reconnaissance. De même, plusieurs épiciers considèrent que ces échanges les enrichissent en tant que personnes et leur font oublier leurs propres problèmes. D'autant plus que mes interlocuteurs sont des immigrés ou issus de l'immigration: leur enracinement territorial, leur intégration dans la ville et dans la société belge passe, dans une large 
mesure, par ce travail. Mais cette condition immigrée, que tous partagent, ne devrait pas non plus renforcer l'idée d'une "communauté de destin » car ces commerçants et leurs employés sont définis par différents statuts sociaux, comme nous le verrons maintenant.

\section{Une « communauté de destin »?}

De manière paradoxale, le métier d'épicier est lié moins à un passé idéalisé qu'à un présent réhabilité : c'est, en grande partie, la généralisation de la grande distribution et des grandes surfaces après la Deuxième Guerre mondiale qui a redoré le blason des épiciers, en leur assignant un rôle valorisé, celui de faire réapparaître le "village ", avec ses solidarités et ses liens forts, en plein milieu de la ville. L'apparition récente des épiceries fines et des commerces de produits-bio a contribué à améliorer leur image.

Comme le souligne Claire Zalc $(2012,57)$, « [r]éalité sociale aux contours flous, le petit patronat se situe dans un intermédiaire, entre le prolétariat et le grand capitalisme. Au début du 20e siècle, artisans, commerçants et petits industriels appartiennent encore au monde ouvrier par leurs pratiques de consommation et de logement ». Au début du 21e siècle, les choses ont considérablement changé : si la plupart des épiciers examinés ici sont issus du monde ouvrier, leur mode de vie ressemble plutôt à celui des classes moyennes - autre catégorie aux contours flous. Tandis que leurs revenus, dans la plupart des cas, les placent largement au sein des classes moyennes, leur origine immigrée et le fait qu'ils exercent un travail qui ne demande pas de qualification ou de diplôme conduisent un certain nombre de leurs clients (surtout dans les quartiers aisés) à les percevoir comme des individus de classe inférieure.

Si la caisse et la balance sont les outils principaux de l'épicier, il serait erroné de l'assimiler à la caissière de supermarché qui manie pourtant les mêmes outils de travail. L'épicier est un travailleur indépendant ${ }^{13}$ et même un petit patron, dans le cas où il emploie des "ouvriers". En règle générale, il existe une continuité dans le commerce de bouche entre le travail salarié et le travail indépendant, dans la mesure où certains employés tentent leur chance et ouvrent leur propre commerce après avoir accumulé la somme d'argent nécessaire et l'expérience ; en revanche, la fermeture d'un commerce peut amener son propriétaire à choisir le « confort » du travail salarié. 
Image 4.

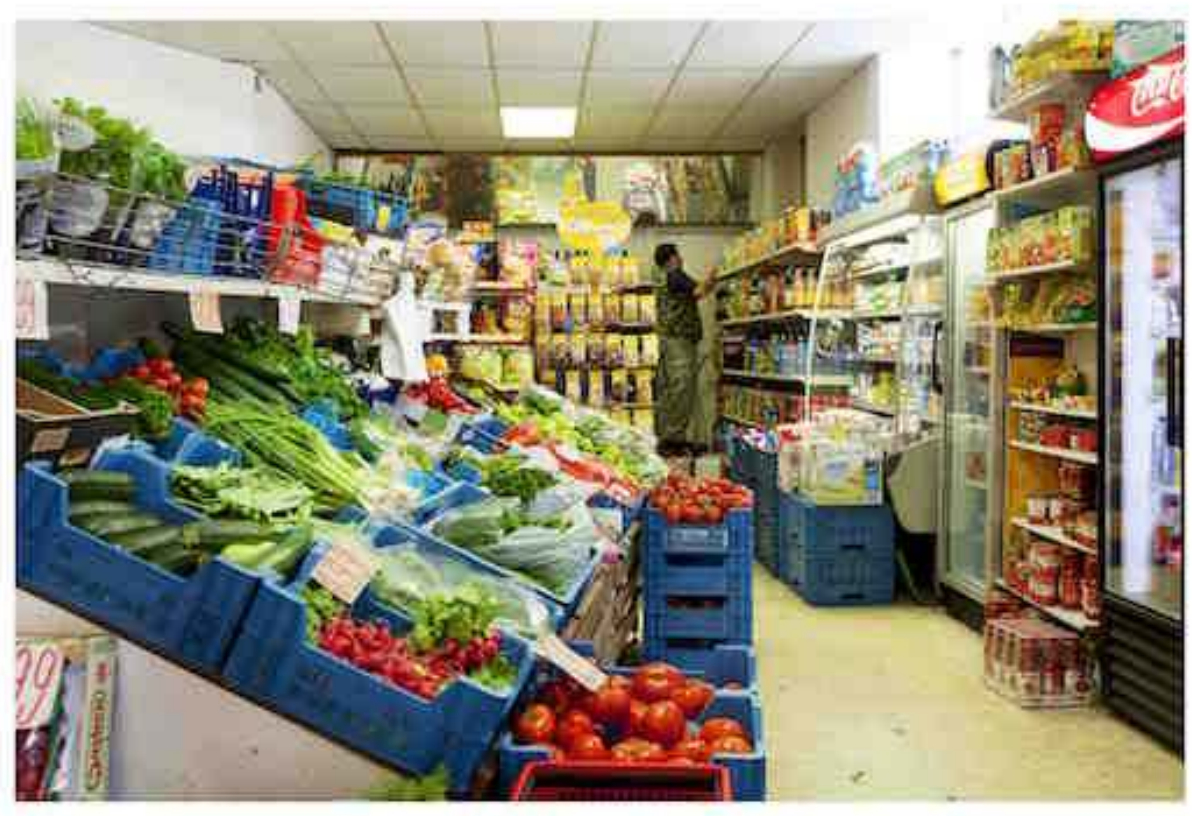

Ce cliché montre un «ouvrier» en train de ranger les rayons au fond du magasin; derrière lui, le diable-chariot l'aide à déplacer la marchandise. En hauteur, le grand miroir permet au propriétaire, qui reste à la caisse, d'avoir une vue panoramique de l'ensemble du local. La caisse reste le centre des opérations et le point de référence, puisque les miroirs ou les caméras de vidéosurveillance sont toujours placés en fonction de son emplacement. Les "ouvriers", auxquels les tâches les plus dures sont déléguées, circulent dans les espaces périphériques (rayons, lieux de stockage, étalages sur le trottoir) et font aussi des livraisons. Leur présence discrète ne doit pas perturber la circulation des clients. Comme le souligne Jean Gadrey $(2005,25)$, « il y a souvent, dans les organisations de service, des ouvriers dont les tâches sont proches de celles de leurs homologues de l'industrie ou du bâtiment ».

Cette image ne montre pas stricto sensu l'épicier ; elle révèle pourtant une partie de son travail, dans la mesure où les commerçants qui n'emploient pas d'«ouvriers » sont obligés d'effectuer toutes ces tâches eux-mêmes. À ce personnage «secondaire » qui occupe une place périphérique (photographié de loin, il ne constitue qu'un détail dans ce "paysage marchand»), s'oppose le portrait d'un autre «ouvrier» qui est placé au cœur de l'image. 
Image 5.

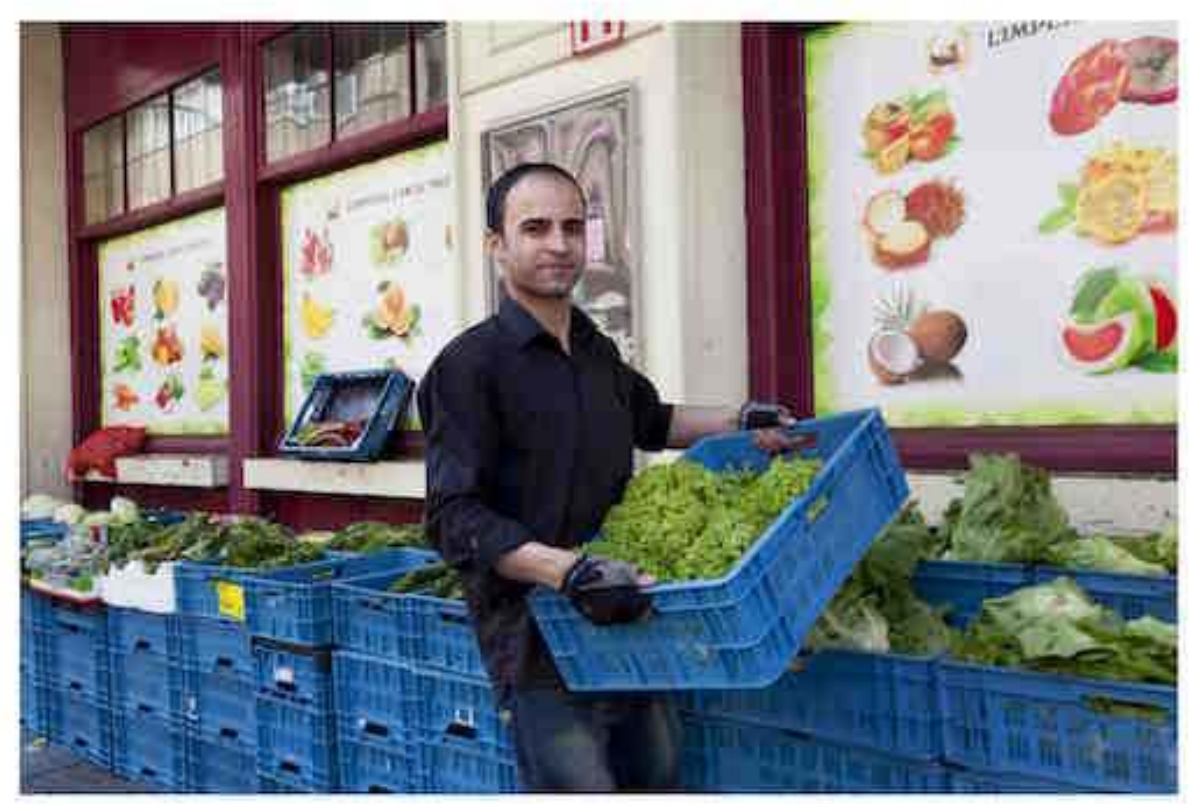

\section{protègent ses mains et diminuent les douleurs musculaires à la fin de la journée, selon son témoignage. S'il est difficile pour ce type d'images de rendre perceptible cette notion abstraite qu'est le travail, il est encore plus compliqué de capter la peine de ces hommes et de ces femmes car la fatigue s'accumule de manière progressive. De la même manière que les couches superposées des habits des épiciers évoquent la pénibilité liée à la position assise et à l'immobilité pendant de longues heures, les gants parviennent à suggérer la pénibilité du travail. Cet accessoire vise autant à optimiser l'efficacité et la performance du corps au travail qu'à le protéger. En effet, le grand nombre de cageots que le cliché capte, ainsi que l'action de l'ouvrier, suggèrent leur déplacement continuel, de l'intérieur à l'extérieur du magasin et vice versa, selon les commandes et les horaires (ouverture et fermeture de l'épicerie).}

La différence vestimentaire entre les deux « ouvriers " photographiés est liée au fait que le second (habillé de manière plus soignée que le premier) tient aussi la caisse, quand l'épicière est absente. La possibilité d'occuper plusieurs postes au cours de la journée et d'être polyvalent crée une certaine confusion, dans la mesure où le client de passage peut facilement se méprendre et penser que cette personne est le propriétaire du magasin. En effet, la distinction entre travailleur indépendant et travailleur subordonné n'est pas toujours évidente. Concernant les salariés, une différence majeure subsiste entre un ouvrier qui tient régulièrement la caisse et celui à qui cette tâche n'est pas confiée. Dans le cas des travailleurs indépendants, il faut distinguer entre :
a. celui qui gère un fond de commerce en partenariat ;
b. celui qui n'a pas de partenaires mais qui paie un loyer, puisqu'il n'est propriétaire que du fond de commerce ;
c. celui qui est propriétaire du local, mais qui ne possède qu'un seul magasin ;
d. celui qui possède plusieurs épiceries. 

l'un à l'autre, comme quand ils ferment une épicerie pour ouvrir un restaurant et d'autre part, les frontières entre ces secteurs restent assez floues. Ainsi, plusieurs épiceries fonctionnent comme des sandwicheries, tandis que certains restaurants, en s'assimilant à des épiceries fines, proposent aux clients des produits-bio de qualité. 
Image 6.

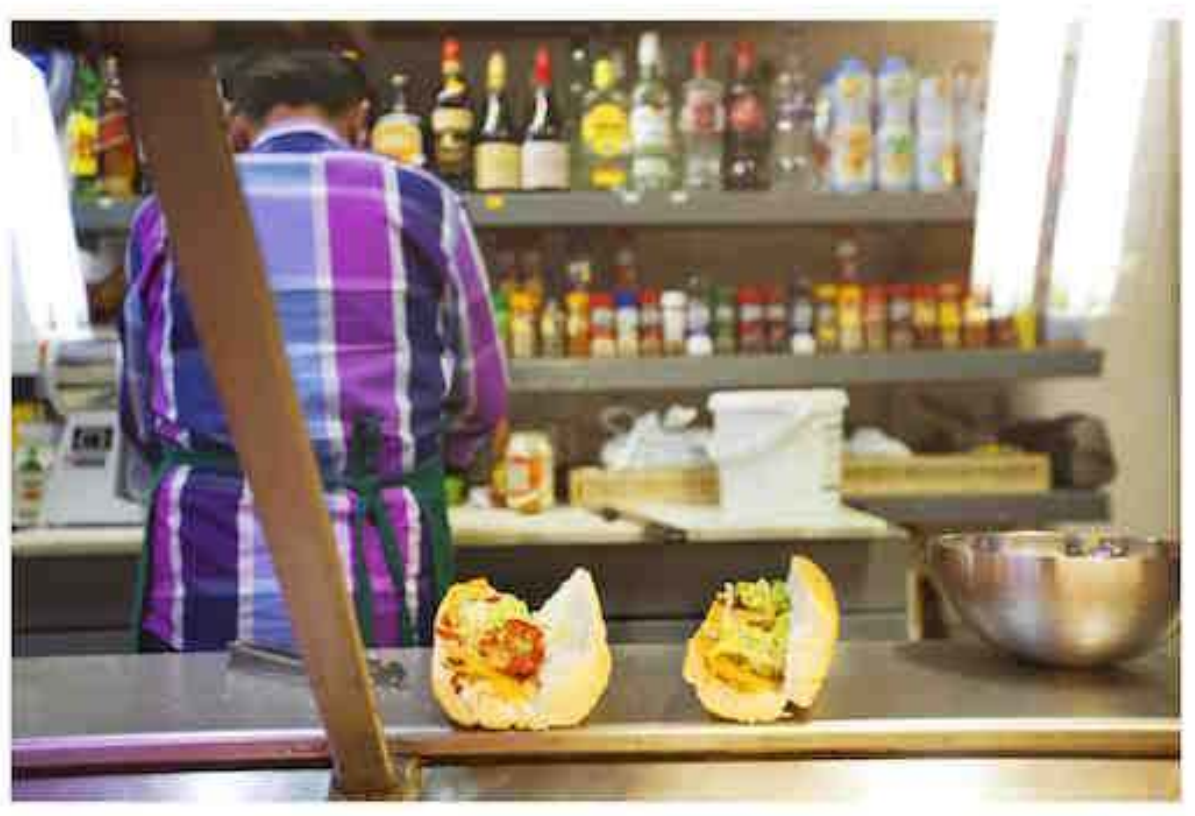

improviste, cette photographie montre le propriétaire dune épicerie en train de préparer deux sandwiches : il utilise comme plan de travail la partie supérieure d'un réfrigérateur. Le cliché est pris à travers les vitres du réfrigérateur, comme les reflets blancs des néons qui apparaissent aux extrémités de l'image le montrent. L'épicier porte un tablier vert qui monte jusqu'à la poitrine (similaire à celui de la femme de la première photographie), mais dont la forme n'est pas vraiment visible, puisqu'il tourne le dos à ses clients mais aussi au photographe et aux spectateurs de cette image. Celle-ci laisse pourtant découvrir d'autres outils que l'épicier utilise de manière récurrente : la pince et le bol au premier plan, puis le couteau et la machine à couper le jambon en arrière plan. En effet, l'épicerie n'est pas seulement un lieu de vente de produits standardisés, dont les emballages fournissent des descriptions incitant à la consommation et soulignant la responsabilité et l'inventivité du fabriquant. Il est aussi un lieu de fabrication, ce qui oblige l'épicier à manier directement des aliments. Dans cet «anti-portrait », les sandwiches et leur matérialité photogénique tiennent le rôle principal: ce produit fini, qui sort des mains de l'épicier, s'oppose au caractère industriel des autres articles en vente. Situés au premier plan, les sandwiches sont nets, contrairement à l'épicier qui est flou et relégué en arrière plan; de plus, l'axe vertical du frigo lui coupe le dos et, concentré comme il l'est sur sa tâche, il tient la tête penchée.

Cette photographie parle aussi de la division de l'espace: mis à part le cliché de l'«ouvrier " au fond du magasin, tous les autres montrent des personnes dont le corps est coupé soit aux hanches soit aux genoux. Ce « morcellement » est, le plus souvent, le résultat de la réticence du photographe à s'introduire dans l'espace qui est réservé à l'épicier. De même, la caisse n'est jamais photographiée depuis l'intérieur. Autrement dit, le photographe qui garde ses distances se place comme un client ordinaire et photographie l'épicier de ce point de vue. 

occasionnellement la caisse, croisent le regard du photographe et, à travers lui, le regard du spectateur de ces images.

Image 7.

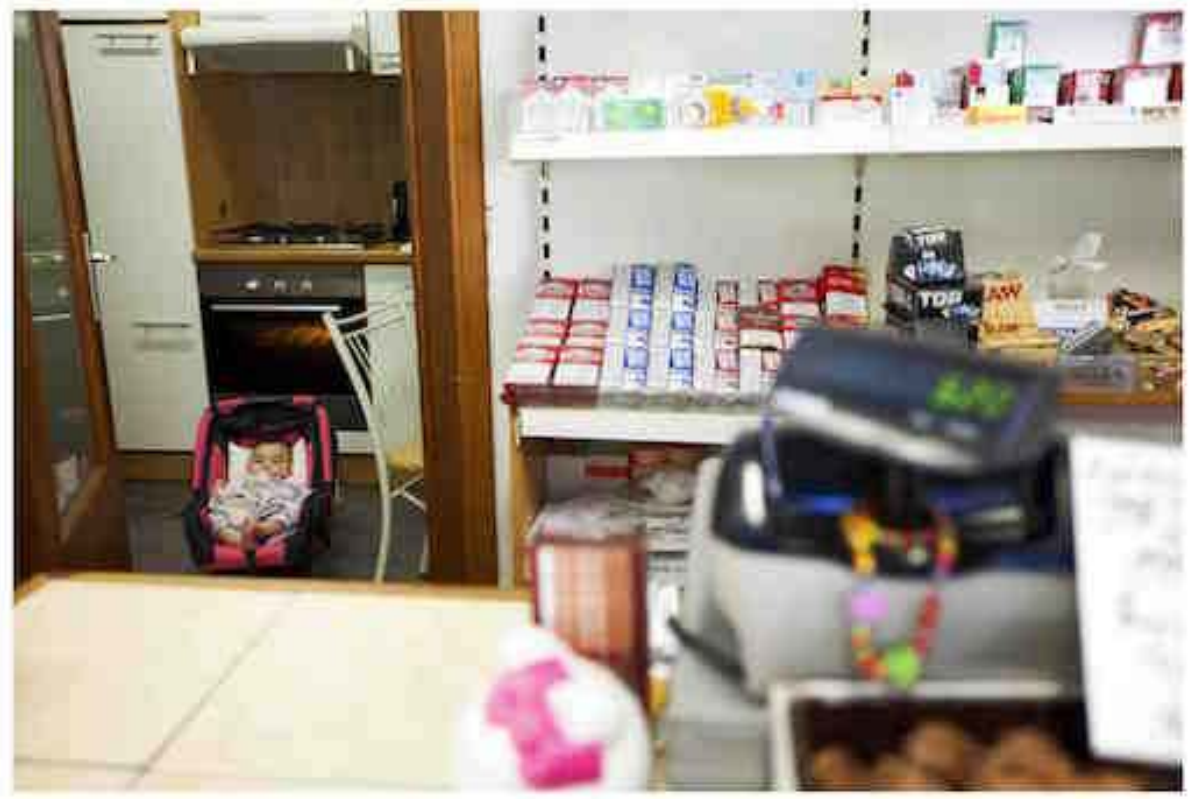

Voilà une autre personne qui rend regard pour regard : le bébé d'une épicière dans sa coque. À l'inverse de la photographie avec les sandwiches, c'est le premier plan qui est flou ici et l'arrière plan qui est net. Le bébé est posé dans une pièce qui se trouve derrière la caisse. Derrière lui, la cuisinière et dans le four, des pains en train de cuire. Dans cette pièce, la propriétaire du magasin (et mère de l'enfant) prépare des feuilletés à la viande et aux légumes qu'elle cuit ensuite au four. La présence du bébé montre ce qu'est le "travail au féminin ", avec ses contraintes et ses compromis. Si le cliché avec l'épicier qui offre une canette à un ami brouille les frontières entre le privé et le public, celui-ci le fait aussi, mais de manière différente, en introduisant une scène de la vie familiale au sein d'un espace marchand. Il montre dans quelle mesure l'épicerie est aussi un lieu de vie, surtout quand les propriétaires n'habitent ni au-dessus du magasin ni même dans le quartier, comme c'est le cas ici. En fait, celui qui regarde cette image peut avoir le sentiment de pénétrer dans la maison de l'épicier, alors qu'il ne voit que l'arrière-boutique. Dans cette pièce qui préserve leur intimité et qui est décorée de manière beaucoup plus personnelle, le jeune couple d'épiciers peut se reposer et se détendre lors des heures creuses.

Tout en refusant de pénétrer dans ces «coulisses " réservées aux commerçants, le cliché laisse entrevoir un espace plus intime ; il révèle ainsi comment vie privée et vie publique s'entremêlent. De la photographie de la façade (dans le cliché de l'ouvrier qui porte un cageot) ${ }^{16}$ jusqu'à celle-ci, la prise de vues parvient à raconter ces variations spatiales, grâce à la juxtaposition.

41 La photographie suivante montre le propriétaire d'un commerce en train de passer la serpillère. 
Image 8.

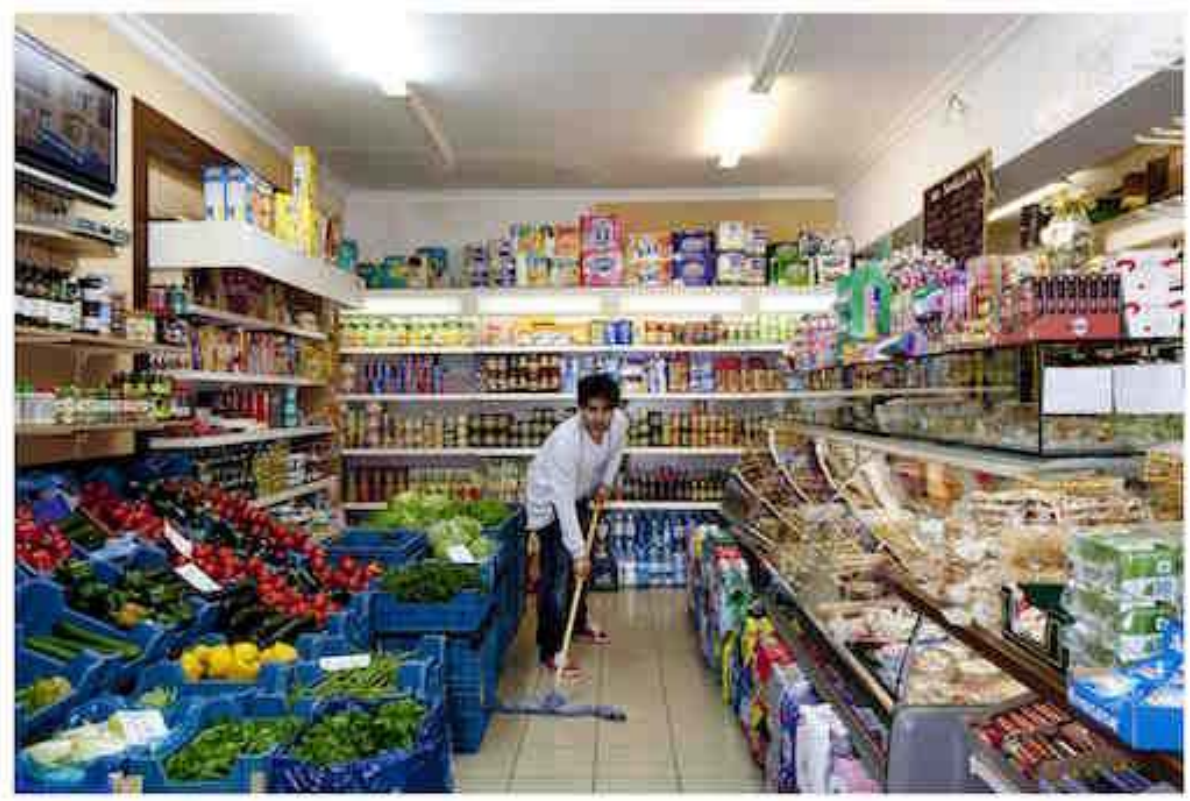

Sur le mur, à droite, on distingue une liste présentant les différents sandwiches, tandis qu'à gauche, en face de la caisse, est installée la télévision qui permet aux commerçants de se détendre quand ils sont moins sollicités par les clients. La serpillère, mais aussi le balai, sont des outils essentiels dans ce type de travail. Ce qui, dans le domaine domestique, correspond à une simple tâche ménagère devient, dans ce cadre marchand, un geste professionnel indispensable, la propreté des lieux étant une condition sine qua non pour la réussite commerciale.

L'épicier est le dernier maillon d'une chaîne qui renvoie tant à la production agricole et artisanale qu'à la fabrication industrielle. Cette chaîne tourne à plein régime grâce à la publicité : chaque article que celui-ci vend fait l'objet d'une campagne publicitaire et est incarné par des visages représentatifs de la clientèle-cible ou choisis pour leur attractivité. L'épicier reste un être invisible dans un univers de marques et de logos qui laisse la publicité assurer sa visibilité. 


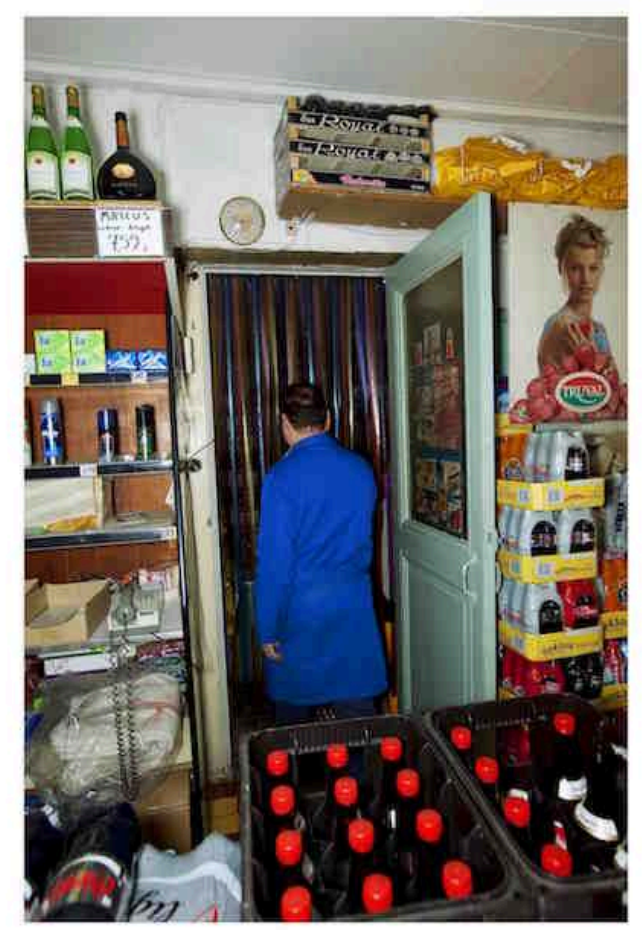
coulisses (l'endroit où il stocke la marchandise), en laissant le photographe et aussi le spectateur de cette image sous le regard de la jeune fille qui pose pour la marque Truval. Il est le seul épicier à porter une blouse bleue qui couvre la totalité du corps. L'espace «privé » vers lequel il se dirige est délimité matériellement par une porte et visuellement par un rideau multicolore qui isole sans empêcher le passage. Là aussi, les frontières entre ce qui est "donné à voir» (car "public») et ce qui fait partie de l'organisation interne de ce lieu de travail sont maintenues.

\section{L'épicerie comme espace photographiable}

Les neuf photographies présentées ici captent une multitude de situations de travail. Une fois mises côte à côte et malgré le fait qu'elles brisent la linéarité du temps et la contiguïté spatiale, elles parviennent à suggérer les ambivalences de ce métier et les variations de situations. Elles nous incitent à passer de l'individuel au collectif, de la spécificité d'un lieu concret à l'épicerie en tant qu'espace générique. Elles ont été choisies pour leur capacité à représenter ce qui est perçu comme l'essentiel du travail : porter et ranger (clichés des deux "ouvriers»), encaisser, préparer des aliments, nettoyer, mais aussi être sociable avec les clients. Bien sûr, il existe d'autres facettes qui ne sont pas représentées, comme la commande au fournisseur ou la tenue des comptes. Chacune d'entre elles constitue une séquence isolée qui fige une partie d'une activité qui est, par définition, continue. Le cliché de l'épicier qui pose avec son ami représente le travail en tant que relation sociale, tandis que les autres insistent plutôt sur des processus techniques. 

sur la profondeur de champ. D'ailleurs, aucune des photographies sélectionnées ne capte les moments d'affluence. Pas de regroupement, pas de photo de couple : ce travail est représenté comme solitaire, même s'il est au centre de la vie familiale et de la sociabilité du quartier. Les clients sont également peu représentés: leur absence est justifiée par les problèmes posés par le droit à l'image, par le souci de produire des images lisibles mais aussi par l'intention de faire émerger l'épicier en tant que personnage central. celui qui montre l'aspect relationnel du travail de l'épicier. Dans les deux cas, ils laissent planer une ombre de confusion - comme si l'épicerie cessait d'être un lieu de travail stricto sensu pendant quelques moments. Selon Philippe Leroux $(1996,134)$, la photographie «ne montre presque pas les formes sociales de l'exécution du travail», c'est-à-dire ce que «le travail vécu [...] a d'abstrait ». Le cliché qui raconte la sociabilité va dans ce sens. De même, Leroux (ibid., 134-135) signale que «[1]a photographie ne manifeste presque pas, dans le travail, l'obligation, sinon la contrainte, quoiqu'elle puisse montrer la misère ou le chômage ». Le cliché avec le bébé parvient à rendre sensible la contrainte, tout en attribuant un rôle social à l'épicière (« mère qui travaille dans une entreprise familiale $»)^{17}$.

Concernant le choix du cadrage, seules deux photographies ont un cadrage vertical : la première pour souligner la proximité entre l'épicier et son client habitué ; la deuxième pour insister sur le contraste et le décalage entre le travail effectif de l'épicier et l'univers de la publicité. Le cadrage vertical a la capacité de rapprocher et de juxtaposer ; en serrant le plan, l'accent est mis soit sur le rapprochement (la sociabilité qui règne dans ces espaces marchands) soit sur la mise à distance (par rapport à la publicité). Ce rapprochement, dans le premier cas, est souligné par le langage des mains qui sont sur le comptoir et se touchent : la canette, qui est posée plus près de la main de l'épicier, constitue l'objet qui les unit, qui fait le lien. D'ailleurs, l'épicier se baisse en s'appuyant sur le comptoir comme pour se mettre à la hauteur de son client, plus petit en taille.

Deux photographies montrent l'épicier de dos. La première, avec son cadrage horizontal, inclut le produit de son travail, le sandwich. La seconde se focalise sur le duel entre l'épicier en tant qu'acteur économique à l'échelle locale et la machine publicitaire. C'est l'acteur local qui ne montre pas son visage, tandis que le visage dominant de la jeune fille sur le poster constitue un commentaire sur l'impersonnalité d'une publicité omniprésente. Si la première photographie constitue un anti-portrait, la seconde s'assimile à une contre-photographie publicitaire.

épiceries sont des lieux de travail complexes. Elles font partie des équipements concurrentiels de la ville, ce qui les oppose aux équipements non concurrentiels (services publics, équipements de santé et d'éducation) ; mais leur caractère en tant que commerces de proximité assigne à ces acteurs économiques des rôles sociaux qui dépassent la sphère économique et qui interrogent tant la notion de «travail relationnel » que la distinction entre commerces et services.

51 Ce reportage ethnographique montre les multiples visages de cet espace de travail qui varient selon le statut de son gérant (partenariat, locataire ou propriétaire, conjoint aidant ou travailleur indépendant) et de ses employés (en relation avec la clientèle ou contraint uniquement aux travaux manuels). Les photographies sont localisées (il s'agit 
des épiceries bruxelloises tenues par des Grecs et des Turcs) et périodisées (elles ont été prises en juin 2013) ; leur singularité est pourtant mobilisée pour conduire jusqu'à la généralisation. Même si l'analyse de ces images instantanées confère aux épiciers photographiés une valeur de représentation qu'ils n'ont jamais revendiquée, elle tente à suggérer derrière chaque séquence, une profession dans sa complexité. La photographie devient alors un "dispositif d'apparition, rendant visible [...] ce qui jusqu'alors était invisible » (Peroni, 1999, 93).

\section{BIBLIOGRAPHIE}

Bourdieu P. (dir.), 1965, Un art moyen. Essai sur les usages sociaux de la photographie, Paris, Les Éditions de Minuit.

Chauchard J.-P. et Le Crom J.-P. (2005), « Les services entre droit civil et droit du travail », Le Mouvement Social, $\mathrm{n}^{\circ}$ 211, p. 51-65.

Collet E. (dir.), 2003, Delhaize « Le lion ». Épiciers depuis 1867, Tournai, Delhaize group.

Colleyn J.-P. (1999), « L'image d'une calebasse n'a pas le goût de la bière de mil. L'anthropologie visuelle comme pratique discursive », Réseaux, 17 (94), p. 19-47.

Conord S. (2007), « Usages et fonctions de la photographie », Ethnologie Française, XXXVII, p. $11-22$.

Du Gay P. (2006), « Le libre-service. La distribution, les courses et les personnes », Réseaux, $\mathrm{n}^{\circ}$ 135-136, p. 33-58.

Gadrey J. (2005), « Un demi-siècle de montée des services : la révolution permanente », Le Mouvement Social, n² 211, p. 21-36.

Lannoy P. (2010), « L'usine, la photographie et la nation. L'entreprise automobile fordiste et la production des photographes industriels ", Genèses, n ${ }^{\circ}$ 80, p. 114-135.

Leroux Ph. (1996), « Quelle photographie du travail ? Quel travail photographié ? », in Peroni M. et Roux J. (dir.), Le travail photographié, Paris, CNRS Éditions-Publications de l'Université de SaintÉtienne, p.127-136.

Papinot Chr. (2007), « Le 'malentendu productif' : Réflexion sur la photographie comme support d'entretien », Ethnologie Française, XXXVII, p. 79-86.

Peyrière M. (2012), « Filmer le travail, filmer contre le cinéma ? », Travailler, $\mathrm{n}^{\circ}$ 27, p. 65-85.

Peroni M. (1999), « Epiphanies photographiques. Sur l'apparition publique des entités collectives », Réseaux, 17 (94), p. 87-128.

Seraïdari K. (2011), « Transactions commerciales et relations sociales entre Grecs et Turcs de Bruxelles », Brussels Studies, [En ligne], 53, consulté le 1 mars 2017. URL : http:// brussels.revues.org/1054

Seraïdari K. (2011-2012), « Paysages marchands en contexte migratoire. Les objets dans les commerces grecs et turcs de Bruxelles ", Études balkaniques. Cahiers Pierre Belon, nº 18, p. 161-178. 
Seraïdari K. (2012), La ville, la nation et l'immigré. Rapports entre Grecs et Turcs à Bruxelles, Paris, L'Harmattan.

Seraïdari K. (2016), « Exotisme, initiatives marchandes et pratiques alimentaires dans les commerces grecs de Bruxelles », Cahiers balkaniques. [En ligne], consulté le 3 avril 2016. URL : http://ceb.revues.org/7115

Zalc Cl. (2012), « Les petits patrons en France au 20e siècle ou les atouts du flou », Vingtième siècle. Revue d'histoire, $\mathrm{n}^{\circ} 114$, p. 53-66.

\section{NOTES}

1. Cinq d'entre elles se situent dans les deux quartiers aisés de Woluwe, tandis que les dix autres se trouvent dans des quartiers intermédiaires ou populaires: trois à Saint-Gilles, deux à Anderlecht, deux à Ixelles et trois à Saint-Josse. Dix de ces magasins appartiennent à des Turcs, deux à des Grecs, deux autres à des Araméens orthodoxes originaires de Turquie, et, enfin, un à une famille kurde de Turquie. Cette collaboration s'inscrit dans le cadre de ma recherche en tant que junior EURIAS fellow, au Vlaams Academisch Centrum de Bruxelles (2012-2013).

2. Ils sont également des commerces de détail et de dépannage, ce qui les distingue des commerces de masse, de gros et de luxe.

3. Ce terme est utilisé de manière récurrente par mes informateurs qui n'emploient pas d'autres qualitatifs pouvant euphémiser ce statut.

4. La volonté de réaliser des entretiens a vite été abandonnée du fait de l'activité incessante des épiciers. Il s'agit donc surtout des prises de notes rapides en présence de l'interlocuteur (commerçant, employé ou client) qui sont complétées par la suite.

5. La réception de l'objet-photographie par les personnes photographiées sera brièvement commentée dans la deuxième section. Les clichés qui leur ont été offerts étaient tous des portraits où elles posaient individuellement ou en couple, et qui se conformaient à «l'esthétique populaire » que Bourdieu analyse (1965, 113-134).

6. Pendant son terrain qui impliquait des prises photographiques, Christian Papinot $(2007,85$, note 4) a constaté que la connaissance « pour la connaissance » ne semble pas être un argument toujours convaincant : en effet, certains de ses interlocuteurs pensaient qu'il réalisait en fait une " étude de marché » afin d'implanter une activité de transport en commun à Madagascar.

7. Ce qui suggère qu'ils ne considèrent la photographie ni comme un miroir réaliste, ni comme l'enregistrement et la documentation du réel. Ils pensent plutôt qu'elle révèle une réalité reconstituée, sublimée et valorisante : la logique publicitaire est donc prédominante, c'est elle qui forme leurs attentes. La troisième phase (qui implique la réception et l'évaluation des photographies par les épiciers) a davantage révélé ces attentes : certains ont remarqué comment l'utilisation d'un appareil « grande angle » change la perception de l'espace, en l'élargissant, en donnant une impression plus spacieuse (et donc flatteuse) de leur commerce. La question de la fictionnalité de la photo préoccupe donc aussi les sujets photographiés.

8. Sur les débuts du libre-service en Grande-Bretagne et les employés de boutique qui considéraient souvent le régime précédent de distribution comme une forme de "servitude", voir Du Gay (2006).

9. Confitures maison ou autres spécialités, qui permettent non seulement d'insister sur la qualité et la fraîcheur de l'offre et de se différencier ainsi des grandes surfaces, mais aussi de recycler des produits périssables et invendus en les cuisinant.

10. Afin de protéger les femmes qui sont longtemps restées invisibles au regard de la loi, la législation belge a défini en janvier 2003 le statut social et fiscal de conjoint(e) aidant(e). Dans un premier temps, cette loi était seulement incitative. En juillet 2005, une nouvelle législation a 
rendu obligatoire de choisir un statut pour le conjoint qui travaille dans une entreprise familiale au moins quatre-vingt-dix jours par an et qui n'a pas d'autre occupation professionnelle. Le statut de conjoint(e) aidant(e) concerne des femmes dans $90 \%$ des cas.

11. Une épicerie spécialisée érige le commerçant en fin connaisseur des produits qu'il vend : en tant qu'expert, l'épicier garantit alors l'efficacité d'un savoir, allant de la sélection des meilleurs produits aux conseils pour leur utilisation et leur conservation.

12. Dans les les épiceries actuelles, le tablier, porté de manière uniforme tant par les femmes que par les hommes, ne participe pas vraiment à la construction d'une identité de genre. Cependant, les photographies de l'épicerie belge Delhaize et de ses succursales qui ont été prises au début du 20e siècle (Collet, 2003) et qui ont été commanditées à des fins publicitaires, montrent que la tenue était un marqueur d'identité sexuelle et sociale. À l'époque, les propriétaires et le personnel sortaient du magasin et posaient à l'entrée de la boutique; sur ces images, les propriétaires se différencient clairement, non seulement parce qu'ils sont au centre de la photographie et devant l'entrée (les employés se rangeant à leurs côtés et occupant des places périphériques), mais aussi parce que leur tenue est différente. Les employés sont les seuls à avoir des blouses qui couvrent la totalité du corps et dont le col est estampillé avec la marque DFC (Delhaize Frères et Cie). De leur côté, les propriétaires portent des tabliers également longs mais qui ne couvrent que la poitrine (les femmes ayant des modèles plus féminins et ouvragés), ainsi que des manchettes de protection pour les avant-bras.

13. Pour protéger les travailleurs indépendants, la législation belge, avec la loi du 25 avril 2007, leur a donné la possibilité de protéger leur résidence principale (insaisissabilité du domicile), s'ils se trouvent submergés par les dettes ou dans une situation de dépôt de bilan.

14. Pour des prises, certains employés ont demandé à être photographiés après s'être assurés de l'accord de leur employeur: l'expérience photographique peut ainsi révéler la nature des rapports entre employeurs et employés.

15. Les services constituent une catégorie difficile à définir. Comme l'expliquent Jean-Pierre Chauchard et Jean-Pierre Le Crom $(2005,51)$, « il n'existe pas de droit du travail spécifique pour les services ». Les auteurs rappellent qu'au 19e siècle les services désignent principalement la classe des domestiques (à laquelle appartiennent aussi les domestiques d'hôtels et les garçons de café), tandis qu'aujourd'hui, par un glissement sémantique, "la notion de service est très valorisée dans le langage managérial » (ibid., 52-53).

16. La façade est l'espace le plus « extérieur » et « public ». Rappelons que l'épicier est un artisan de l'espace urbain, dans la mesure où ces magasins peuvent être un facteur de revitalisation urbaine ou même fonctionner comme des forces d'attraction conduisant à la gentrification d'un quartier.

17. Il fait aussi allusion à une histoire personnelle spécifique, puisque le bébé incarne la quatrième génération impliquée dans la vie de l'épicerie (les parents et les grands-parents de la mère étant eux-aussi des épiciers dans d'autres quartiers de la ville). Le bébé, qui est déjà familier avec ce lieu de travail, devient ainsi le témoin d'une continuité, ce que seule l'enquête ethnologique peut saisir.

\section{RÉSUMÉS}

Cet article examine comment un corpus photographique permet d'éclaircir les multiples facettes du travail d'épicier. Dans ce cadre, la photographie, en tant que « dispositif d'apparition », fait 
émerger derrière chaque geste, une profession dans sa complexité. L'analyse iconographique montre ainsi la place que ces acteurs économiques occupent à l'échelle du quartier, mais aussi au sein de l'espace urbain: leur rôle dépasse souvent la sphère économique et interroge tant la notion de « travail relationnel » que la distinction entre commerces et services.

This article examines how a photographic corpus allows to better understand the multifaceted nature of grocer's work. In this framework, photography, that functions as "a mechanism of apparition", brings to light behind every move, a profession and its complexity. The iconographic analysis reveals thus the place that these economic actors occupy in the neighbourhood, but also in the urban space: their role, which often goes beyond the economic sphere, interrogates the notion of "relational work", but also the distinction between boutiques and services.

\section{INDEX}

Mots-clés : épicerie, travail relationnel, services, quartier, Bruxelles

Keywords : grocery, relational work, services, neighbourhood, Brussels

\section{AUTEUR}

\section{KATERINA SERAÏDARI}

Docteur en anthropologie sociale depuis 2000 (EHESS-Toulouse), Katerina Seraidari est membre associée du LISST-Centre d'Anthropologie Sociale à Toulouse. En 2009-2010, grâce au financement de l'Institut d'encouragement de la Recherche Scientifique et de l'Innovation à Bruxelles (IRSIB), elle a commencé une nouvelle recherche sur les commerces grecs et turcs de Bruxelles. En 2012-2013, en tant que junior EURIAS fellow à l'Académie flamande (VLAC), elle a prolongé cette recherche, se concentrant cette fois sur les épiceries grecques et turques de Bruxelles. Elle est l'auteur de trois livres : Le culte des icônes en Grèce, Toulouse, Presses Universitaires du Mirail, 2005 ; «Que sa grâce soit grande! » Pratiques dévotionnelles et conflits idéologiques dans les Cyclades, Athènes, Erinni-Philippotis, 2007, publié en grec ; et, enfin, La ville, la nation et l'immigré. Rapports entre Grecs et Turcs à Bruxelles, Paris, L'Harmattan, 2012. Ce livre a reçu le prix Adelson Castiau 2015 de l'Académie royale des sciences, des lettres et des beaux-arts de Belgique. 